\title{
Quantitative Studies of the Local Exudative Cellular Reaction in Acute Leukemia*.
}

\author{
Pasquale E. Perillie and Stuart C. Finch \\ (From the Medical Service, Veterans Administration Hospital, West Haven, and the De- \\ partment of Internal Medicine, Yale University School of Medicine, New \\ Haven, Conn., and the Grace-New Haven Community Hospital)
}

The quantitative evaluation of local cellular response to an inflammatory stimulus in patients with acute leukemia is described in this report. A special glass chamber was used to collect leukocytes that accumulated at an exudative forearm lesion over a period of 24 hours in patients with acute leukemia and their controls. The total cellular response for each subject was based on direct leukocyte count of the fluid exudate samples collected in the glass chamber.

Previous study of the local exudative reaction to inflammation with the coverslip method of Rebuck suggests that the acute cellular response in patients with acute leukemia is retarded in onset and diminished in intensity (1-3). The Rebuck method, however, is not quantitative, and the magnitude of the changes in acute leukemia has been difficult to determine. Because of the importance of the acute cellular response in host defense against microbial invasion, the chamber technique was developed to determine whether the previous impression is supported by actual inflammatory cell counts.

\section{Materials and Methods}

Twelve forearm leukocyte mobilization tests were performed on 10 adult control subjects. Nine of the 10 were male, ranging in age from 24 to 63 years. The control subjects consisted of hospitalized patients convalescing from either mild neurologic or vascular illness. At the time of study none had evidence of hematologic disease, laboratory or clinical evidence of infection, or was receiving any medication that was known to affect the dynamics of the inflammatory response.

Fifteen forearm leukocyte mobilization tests were performed on a group of thirteen adult patients with acute leukemia. The age distribution of these patients was

* Submitted for publication July 3, 1963; accepted November 8, 1963.

Supported by the Veterans Administration and U. S. Public Health Service grant HE 07616-01. comparable to that of the control subjects. Each was studied just before the initial administration of antileukemia therapy. Three of the patients with leukemia had acute infections at the time of the study and were receiving antimicrobial therapy. In two instances, the study was repeated before the institution of antileukemia therapy.

The initial step in the technique for performing the chamber leukocyte mobilization test involved producing a single, uniform, 2-cm-square, abraded lesion on the volar surface of the forearm of the nondominant side of each patient (4). The lesion was covered immediately with a specially designed glass cup chamber (Figure 1 ). The chamber was made of Pyrex glass, measured $2.5 \mathrm{~cm}$ in diameter and $2 \mathrm{~cm}$ in height, and had a total capacity of $4.5 \mathrm{ml}$. On opposite sides of the chamber, separate glass spouts had been attached so that one outlet communicated with the upper level of the chamber cavity and the other with the lower level. Glass hooks also were located on the sides between the spouts to anchor the chamber to the forearm with a snug, but not tightfitting rubber band $5 \mathrm{~cm}$ wide. A 2-cm piece of sterile plastic tubing connected each spout with a 3-way stopcock.

The chamber was filled and emptied as illustrated in Figure 2. A sterile $10-\mathrm{ml}$ syringe (B) containing $8 \mathrm{ml}$ of buffered, isotonic saline $(\mathrm{pH} 7.4)$ and $1,000 \mathrm{U}$ of Varidase (streptokinase and streptodornase) was attached to the lower spout via the 3-way stopcock and plastic tubing. A sterile, empty $10-\mathrm{ml}$ syringe (A) with identical connections was attached to the upper spout. The chamber was filled with buffered saline by applying gentle traction to the plunger of the empty syringe (B). At this point, the previously empty syringe usually contained about $3 \mathrm{ml}$ of overflow fluid. Both stopcocks were closed, the syringes were removed, and adhesive tape was used to secure additional fixation of the chamber unit to the forearm. The patient then was permitted free use of the arm.

Two to 3 hours after the initial inflammatory stimulus the chamber was emptied in the following manner: A sterile 50-ml syringe (A) containing buffered isotonic saline was attached to the upper spout and a sterile empty 10-ml syringe (B) to the lower spout. The chamber was emptied by pulling the fluid into the empty lower syringe. Ten washings of $5 \mathrm{ml}$ each were collected and transferred into separate graduated centrifuge tubes. All collections were centrifuged at 3,000 


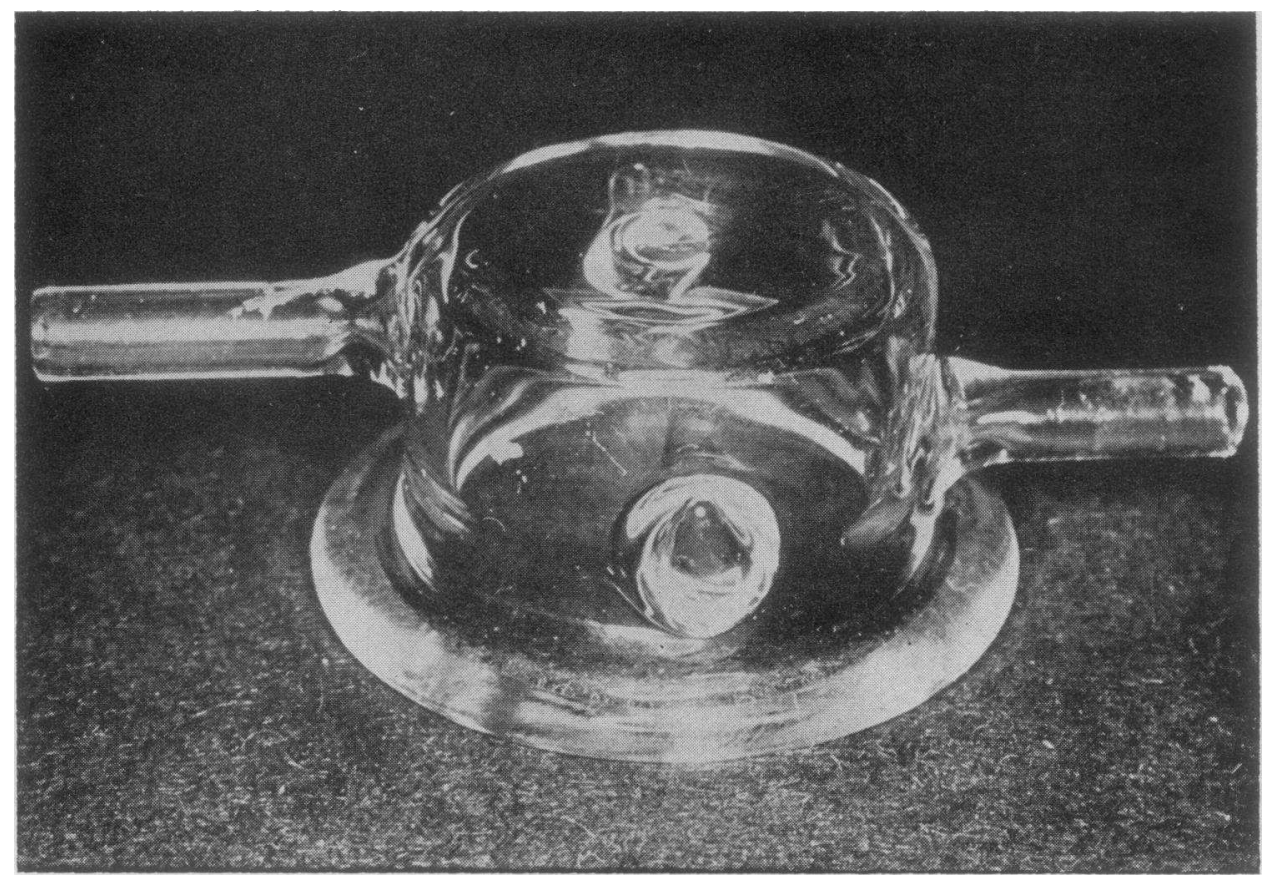

Fig. 1. Photograph of CUP Chamber EMPloyed in PRESENT STLDY.

rpm for 30 minutes. All but $0.5 \mathrm{ml}$ of the supernatant fluid was discarded. The cellular sediment was resuspended in the $0.5 \mathrm{ml}$ of remaining supernatant fluid, and total leukocyte counts were performed in a hemocytometer using $2 \%$ acetic acid as the diluent. In some instances, differential counts were performed on smears made from the recentrifuged leukocyte suspensions.

The chamber was refilled and emptied as described above 5 to 6 hours and 24 hours after the original in- flammatory stimulus. At 24 hours the chamber was removed and the surface of the lesion gently scraped with a sterile 20 -gauge needle. The needle was shaken briskly into a centrifuge tube containing buffered saline. Total and differential leukocyte counts were performed on the centrifuged sediment as described above.

Statistical comparisons of the total cellular collections of each group were carried out by a modified $t$ test, and significance was reported in terms of $p$ value.

\section{METHOD OF FILLING AND EMPTYING FOREARM CHAMBER}

\section{FILLING}

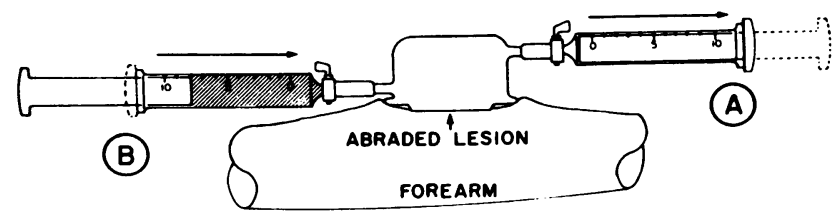

\section{EMPTYING}

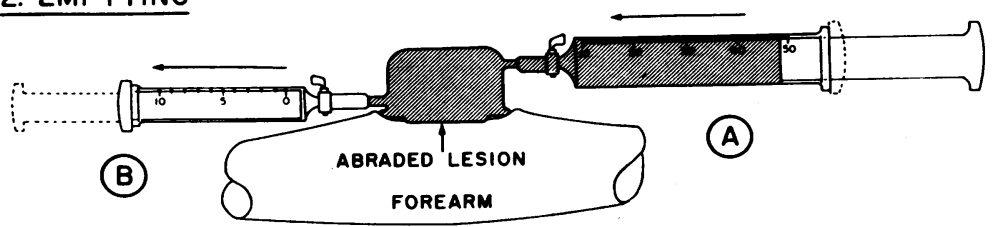

Fig. 2. Diagram of METHOD FOR FILLING AND EMPTying ChAMber (see text for details). 
TABLE I

Control patients

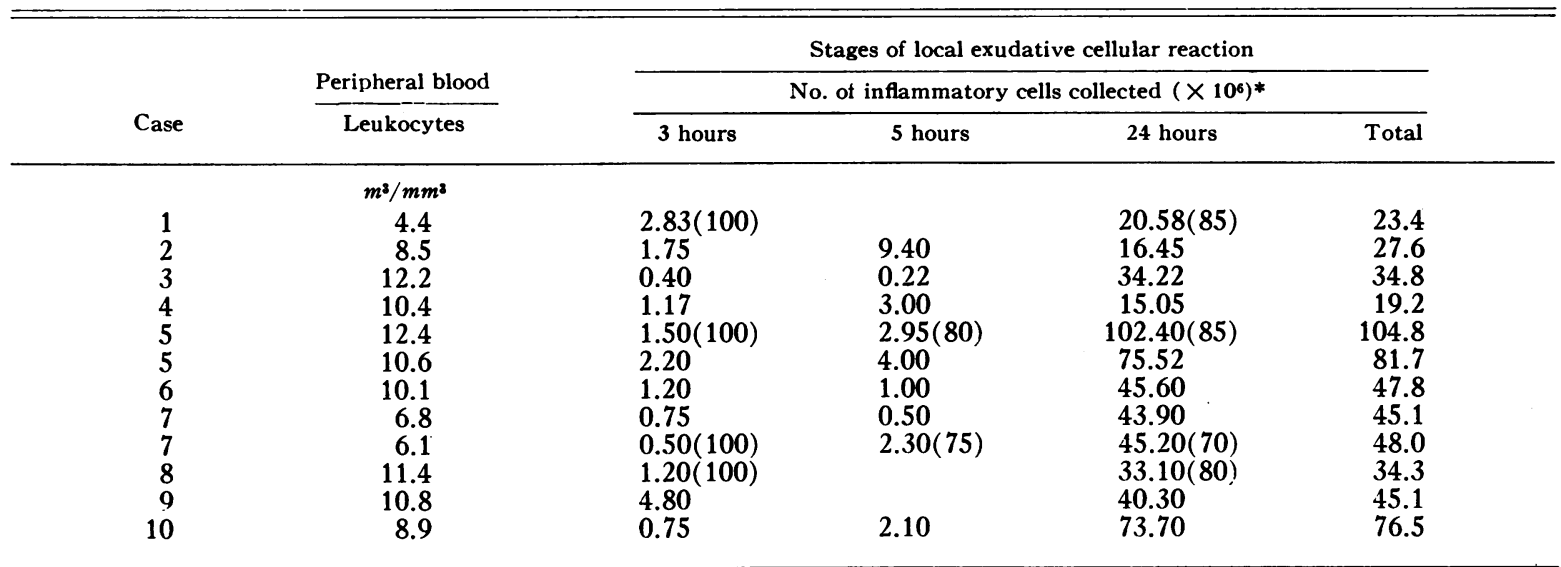

* Figures in parentheses represent the percentage of mature granulocytes in the exudative reaction ( 500 cells counted).

\section{Results}

Control subjects (Table I). In 8 of 10 tests, more than 1 million cells were collected during the first 3 hours of the inflammatory reaction with the range being from 1.17 to 2.83 million cells. In the 8 tests measured at the 5- to 6-hour stage, 6 had total cellular collections of 2 million or greater. The total 24-hour accumulation was greater than 20 million cells in 11 of the 12 tests performed and averaged 49.2 million cells for all tests performed within the group.
The chamber studies were repeated in two control subjects (Subjects 5 and 7, Table I) on separate days to determine the reproducibility of the test. There was close agreement between the results of the separate tests at all stages of the inflammatory cycle. Differential counts on the chamber leukocytes during the first few hours showed the entire cellular accumulation to consist of segmented and band polymorphonuclear leukocytes. Between the third and sixth hours a few lymphocytoid cells appeared, although segmented polymorphonuclear leukocytes continued

TABLE II

Acute leukemia patients

\begin{tabular}{|c|c|c|c|c|c|c|c|c|c|}
\hline \multirow{3}{*}{ Case } & \multirow{3}{*}{ Type of leukemia } & \multicolumn{2}{|c|}{ Peripheral blood } & \multirow{2}{*}{\multicolumn{6}{|c|}{$\frac{\text { Stages of local exudative cellular reaction }}{\text { No. of inflammatory cells collected }\left(\times 10^{6}\right) \dagger}$}} \\
\hline & & \multirow{2}{*}{ Leukocytes } & \multirow{2}{*}{$\frac{\text { Polys* }}{\text { Blasts }}$} & & & & & & \\
\hline & & & & 2 hours & 3 hours & 5 hours & 6 hours & 24 hours & Total \\
\hline & & $10^{3} / \mathrm{mm}^{2}$ & $\%$ & & & & & & \\
\hline 1 & Granulocytic & 182.0 & $10 / 40$ & 0 & $1.20(100)$ & & $0.80(80)$ & $1.50(75)$ & 3.5 \\
\hline 1 & Granulocytic & 165.0 & $15 / 30$ & 0 & $1.40(100)$ & & 1.20 & $1.60(75)$ & 4.2 \\
\hline 2 & Monocytic & 208.0 & $20 / 20$ & & 0 & & 0.60 & 0.40 & 1.0 \\
\hline 2 & Monocytic & 225.0 & $10 / 28$ & 0 & 0 & & $2.40(75)$ & $3.40(85)$ & 5.8 \\
\hline 3 & Monocytic & 39.2 & $30 / 20$ & & 0 & & 0.10 & 13.70 & 13.8 \\
\hline 4 & Monocytic & 90.1 & $15 / 30$ & & 0 & & 1.40 & 1.30 & 2.7 \\
\hline 5 & Granulocytic & 33.5 & $1 / 61$ & & 0.50 & & 0.10 & 7.00 & 7.6 \\
\hline 6 & Granulocytic & 90.0 & $0 / 40$ & & 0.15 & & $0.15(90)$ & $10.90(85)$ & 11.2 \\
\hline 7 & Granulocytic & 19.6 & $30 / 20$ & & $0.10(100)$ & & 0.15 & $0.75(80)$ & 1.0 \\
\hline 8 & Granulocytic & 13.6 & $10 / 15$ & & 0.15 & 0.62 & & 4.10 & 4.9 \\
\hline 9 & Granulocytic & 3.7 & $0 / 70$ & & 0.20 & & $0.20(85)$ & $1.70(80)$ & 2.1 \\
\hline 10 & Lymphocytic & 50.4 & $40 / 21$ & & 0.05 & 1.60 & & 30.50 & 32.1 \\
\hline 11 & Lymphocytic & 3.6 & $10 / 50$ & 0 & & & 0.40 & 0.90 & 1.3 \\
\hline 12 & Stem cell & 4.7 & $10 / 40$ & 0 & & 0.37 & & 18.80 & 19.1 \\
\hline 13 & Stem cell & 56.1 & $51 / 19$ & & $0.02(100)$ & & 0.02 & 17.50 & 17.5 \\
\hline
\end{tabular}

* Polymorphonuclear leukocytes/blasts.

$\dagger$ Figures in parentheses represent the percentage of mature granulocytes in the exudative reaction (500 cells counted). 
to predominate. At 24 hours appreciable numbers of macrophages were present, but again segmented and band polymorphonuclear leukocytes were the major cell types. At all stages of the exudative reaction, qualitative leukocyte responses in the control subjects were very similar (Table I).

Leukemia patients (Table II). In 11 of 13 tests performed on the leukemia patients, fewer than 500,000 cells were collected during the first 3 hours of the inflammatory reaction (Table II). In four cases no cells were present in the fluid collected at 3 hours. In only 3 of 15 tests were more than 2 million cells collected by the sixth hour. The 24-hour total collection was less than 8 million cells in 10 of 15 tests and was less than 20 million in 14 of the 15 tests performed. The average 24-hour collection in this group was 8.52 million cells. Duplicate tests on two patients again showed relatively close agreement, especially in regard to the total cellular accumulation at 24 hours (Patients 1 and 2; Table II). Analysis of the means of the total cellular collections in each group showed a differential between the control and leukemia group that was significant $(\mathrm{p}<0.001)$.

Differential counts on the chamber leukocyte suspensions were very similar to those from the control subjects. Segmented and band polymorphonuclear leukocytes predominated at all stages, but some lymphocytoid forms and macrophages appeared during the later hours of the reaction. No blast forms were found on the coverslip smears from any of the leukemia patients at any stage of the exudative reaction.

\section{Discussion}

The results of the forearm chamber study indicate that the local exudative cellular response in adult patients with untreated acute leukemia is quantitatively deficient when compared to hematologically normal subjects. In the leukemia group the number of exudative leukocytes remained uniformly reduced regardless of age, sex, morphologic type of leukemia, or percentage of immature leukocytes in the peripheral blood. Failure of these patients to mobilize cells under these experimental conditions suggests that the local cellular response in adult patients with acute leukemia in relapse may be inadequate to prevent more widespread dissemination of local bacterial infection.

Determining the error of the chamber method has been impossible, but the technique probably does not permit complete recovery of cells present at the foci of induced inflammation. Nevertheless, in the present comparative study it seems unlikely that errors of collection alone could have accounted for the marked differences between the total inflammatory cells collected in the control and leukemia groups. The near agreement of results in repeated determinations in individual patients suggests that the method when properly performed will give reproducible results.

In performing the forearm abrasion, care was exercised to ensure that the lesion was of uniform size and depth. This was best controlled by gentle abrasion of a carefully measured 2-cmsquare area of forearm skin until fine bleeding points appeared, a reliable sign that the papillary layer of the dermis had been exposed. Too vigorous abrasion usually led to brisk bleeding and thereby rendered the lesion unsuitable for study. When the abrasion was properly performed, the fluid subsequently removed from the chamber was free of red cells.

The method of emptying and flushing the chamber was carefully standardized, even though preliminary studies indicated that variations in the amount of traction applied to the plunger of the lower syringe had little influence on the number of cells collected. During emptying of the chamber, negative pressure was considered too great if the surface of the abraded skin became elevated.

Occasionally leakage of chamber fluid beneath the edge of the chamber was a problem. Usually this was due to the development of furrows between the flexor muscles of the forearm during forceful flexion of the hand and wrist. This problem was avoided by placing the abraded lesion more proximally on the forearm.

Varidase was added to the chamber fluid to prevent the mobilized leukocytes from clumping together and sticking to the surface of the abraded skin. That the Varidase prevented excessive fibrin deposition and leukocyte trapping is likely. The Varidase also may have enhanced the cellu- 
lar inflammatory response and may have been responsible for the persistence of polymorphonuclear leukocytes during the later stages of the exudative reaction. Varidase had not been used previously with the coverslip method, and late cellular accumulations contained relatively more macrophages (2).

Several of the patients with acute granulocytic leukemia (Patients 5, 6, and 9; Table II) had virtually no mature polymorphonuclear leukocytes in their peripheral blood smears. Their exudative cellular reactions, although quantitatively deficient, were almost entirely polymorphonuclear in type. Jaffé has suggested that patients with acute leukemia will respond to inflammation with morphologically mature cells as long as some portion of the marrow remains functionally unimpaired. In the absence of any remaining foci of normal myeloid precursors, he was unable to demonstrate an inflammatory response (5). Our patients with this paradoxical reaction must have had foci of functionally normal marrow and must have maintained a small noncirculating pool of available mature granulocytes.

The total number of cells mobilized during the exudative cellular reaction in acute leukemia is not determined solely by the number of mature cells in circulation. In some instances the absolute number of mature granulocytes in the peripheral blood (Patients 1, 2, 4, and 7; Table II) was increased, yet the total number of cells mobilized in the chamber was greatly reduced. This reduction in polymorphonuclear leukocyte migration may be the result of a small reserve pool, leukocyte functional impairment, or adverse extrinsic influences. Additional investigation will be necessary to clarify this point.

Resistance to infection of patients with acute leukemia while in remission is essentially normal. Reduced resistance is most strikingly seen in patients in relapse. Unfortunately, in the present study we were unable to re-examine the two patients who obtained good responses to treatment during remission. These two patients (Patients 10 and 12; Table II) were, however, the only leukemia patients whose total cellular responses fell within the normal range. This may have been fortuitous, but it is tempting to suggest that a favorable response to chemotherapy may depend upon having residual marrow foci capable of providing the body with an adequate supply of functionally intact inflammatory cells. Other interpretations are possible, but this relationship deserves additional study.

No consistent abnormalities in antibody formation, properdin levels or leukocyte phagocytosis have been demonstrated in patients with acute leukemia (6-10). Several experimental studies, however, have now demonstrated defects in the inflammatory cellular response of such patients. The investigations of both Boggs and Riis, employing the coverslip method for study of the local exudative reaction, found that the quantity of cells in exudates of patients with acute leukemia usually was markedly reduced, and not infrequently, was acellular $(1,3)$. In a similar type of study we also observed that some acute leukemia patients showed no exudative cellular reaction to local inflammatory stimulation (2). In addition, we found a delay in the initial onset of the cellular inflammatory response. However, ascertaining the extent of these changes on coverslip smears was difficult, but the chamber studies did demonstrate defects in both the temporal and quantitative aspects of the local cellular response.

Animal studies have shown the early cellular response to be the most important determinant of whether cutaneous bacterial infection will be able to progress (11). Other deficiencies in the defense mechanisms of the acute leukemic host probably exist, but failure to mobilize normal numbers of acute inflammatory cells rapidly suggests that this may represent a serious defect in leukemic host resistance to bacterial infection. One would expect that such a defect would predispose to enhance microbial invasion of the body. That bacterial infections in patients with acute leukemia tend to disseminate more rapidly than those of otherwise normal subjects is well recognized. Four of the patients with leukemia included in this study developed terminal gramnegative septicemias.

Further studies of the inflammatory cellular response in other disorders characterized by increased susceptibility to infection are indicated. The method employed in the present study is particularly suited for this purpose, since it provides a more quantitative and objective com- 
parison of the exudative cellular reaction in humans than has heretofore been possible.

\section{Summary}

The local exudative cellular response was studied by means of a quantitative chamber technique in thirteen patients with untreated acute leukemia and in ten control subjects. There was significant reduction in the total number of leukocytes mobilized by the leukemic group during the 24-hour period of study. These results suggest that patients with acute leukemia may mobilize leukocytes poorly at sites of local infection and that they may represent a serious defect in their resistance to certain kinds of infection.

\section{References}

1. Riis, P. The Cytology of Inflammatory Exudate. Copenhagen, Munksgaard, 1959.

2. Perillie, P. E., and S. C. Finch. The local exudative cellular response in leukemia. J. clin. Invest. 1960, 39, 1353.

3. Boggs, D. R. The cellular composition of inflam- matory exudates in human leukemias. Blood 1960, 15, 466.

4. Rebuck, J. W., and J. H. Crowley. A method of studying leukocyte functions in vivo. Ann. N. Y. Acad. Sci. 1955, 59, 757.

5. Jaffé, R. H. Morphology of the inflammatory defense reactions in leukemia. Arch. Path. 1932, 14, 177.

6. Larson, D. L., and L. J. Tomlinson. Antibody response in leukemia. J. clin. Path. 1953, 32, 317.

7. Silver, R. T., J. P. Utz, J. Fahey, and E. Frei III. Antibody response in patients with acute leukemia. J. Lab. clin. Med. 1960, 56, 634.

8. Hinz, C. F., Jr. Properdin levels in infections and noninfectious disease. Ann. N. Y. Acad. Sci. 1956, 66, 268.

9. Silver, R. T., G. A. Beal, M. A. Schneiderman, and N. B. McCullough. The role of the mature neutrophil in bacterial infections in acute leukemia. Blood 1957, 12, 814.

10. Braude, A. I., J. Feltes, and M. Brooks. Differences between the activities of mature granulocytes in leukemic and normal blood. J. clin. Invest. 1954, 33, 1036.

11. Miles, A. A., E. M. Miles, and J. Burke. The value and duration of defense reactions of the skin to the primary lodgement of bacteria. Brit. J. exp. Path. 1957, 38, 79. 